\title{
Accurate calculation of the complex eigenvalues of the Schrödinger equation with an exponential potential
}

\author{
Paolo Amore ${ }^{1}$ \\ Facultad de Ciencias, Universidad de Colima, Bernal Díaz del Castillo 340, \\ Colima, Colima, Mexico \\ Francisco M. Fernández ${ }^{2}$ \\ INIFTA (Conicet, UNLP), División Química Teórica, \\ Diag. 113 y 64 (S/N), Sucursal 4, Casilla de Correo 16, \\ 1900 La Plata, Argentina
}

\begin{abstract}
We show that the Riccati-Padé method is suitable for the calculation of the complex eigenvalues of the Schrödinger equation with a repulsive exponential potential. The accuracy of the results is remarkable for realistic potential parameters.
\end{abstract}

\section{Introduction}

Recently, Rakityansky et al [1] developed a method for the calculation of bound states and resonances of quantum-mechanical problems. It is based

$\overline{1}$ paolo.amore@gmail.com

2 e-mail: fernande@quimica.unlp.edu.ar (corresponding author) 
on the approximation of the S-matrix by means of Padé approximants and the location of their poles. The authors mention that the methods for the calculation of bound states and resonances are usually developed separately in spite of the fact that those quantities share the same mathematical nature.

Some time ago we developed the Riccati-Padé method that applies to bound states and resonances of separable quantum-mechanical models [2-11]. Although the RPM is less general than the approach proposed by Ratikyansky et al [1] it is nevertheless an interesting approach for comparison purposes and benchmark.

The RPM [2-11] is based on a rational approximation to a modified logarithmic derivative of the wavefunction that satisfies a Riccati equation. From the coefficients of the Taylor expansion of this logarithmic derivative, which are functions of the energy, we construct Hankel determinants. Their roots give rise to sequences that converge towards the bound states and resonances of the quantum-mechanical model as the determinant dimension increases. In most cases the rate of convergence is so great that the RPM yields extremely accurate real and complex eigenvalues.

The rational approximation and the Hankel quantization condition appear to select square integrable functions and incoming or outgoing waves. Both, bound states and resonances emerge from the Hankel sequences because one does not introduce the boundary conditions at infinity explicitly.

Earlier results for exponential potentials suggest that the RPM may not yield virtual states and that the Hankel sequences converge to a wrong limit, although, suspiciously close to the right answer, in the case of some resonances [9]. The purpose of this paper is to investigate this feature of the RPM more closely.

In Section 2 we discuss a simple model that enables us to calculate the poles 
of the scattering amplitude from the roots of Bessel functions. In Section 3 we apply the RPM to this model and compare approximate and exact results. Finally, in Section 4 we summarize our results and draw conclusions.

\section{Model}

In this paper we test the performance of the RPM on the Schrödinger equation

$$
\psi^{\prime \prime}(x)+\left(E-A e^{-\alpha x}\right) \psi(x)=0
$$

with the boundary condition $\psi(0)=0$. This model has proved useful in the past for the study of bound, resonance, and virtual states [12-14]. Besides, the exponential potential is a suitable representation of repulsive molecular interactions [13]. The change of variables $q=\alpha x, \Phi(q)=\psi(q / \alpha)$ leads to an eigenvalue equation with just one potential parameter:

$$
\Phi^{\prime \prime}(q)+\left(\epsilon-\lambda e^{-q}\right) \Phi(q)=0
$$

where $\epsilon=E / \alpha^{2}$ and $\lambda=A / \alpha^{2}$.

A further change of variables $z=2 \sqrt{-\lambda} e^{-q / 2}, Y(z)=\Phi(2 \ln [2 \sqrt{-\lambda} / z])$ transforms the eigenvalue equation (2) into the Bessel equation

$$
z^{2} Y^{\prime \prime}(z)+z Y^{\prime}(z)+\left(z^{2}-\nu^{2}\right) Y(z)=0
$$

where $\nu^{2}=-4 \epsilon$. The general solution that satisfies the boundary condition at $q=0$ is $[12]$

$$
Y(z)=C\left[J_{-\nu}(2 \sqrt{-\lambda}) J_{\nu}(z)-J_{\nu}(2 \sqrt{-\lambda}) J_{-\nu}(z)\right]
$$

If we assume that the RPM will provide the eigenvalues of those solutions that satisfy $\lim _{q \rightarrow \infty} \Phi(q)=0$, then it follows from the behaviour of the Bessel 
function at origin $J_{\nu}(z) \sim(z / 2)^{\nu} / \Gamma(\nu+1)$ that they should be roots of

$$
J_{\nu}(2 \sqrt{-\lambda})=0
$$

with $\operatorname{Re}(\nu)>0$. The roots of this equation are poles of the scattering amplitude [13].

The modified logarithmic derivative of an eigenfunction of equation (2)

$$
f(q)=\frac{1}{q}-\frac{\Phi^{\prime}(q)}{\Phi(q)}
$$

satisfies the Riccati equation

$$
f^{\prime}(q)+\frac{2 f(q)}{q}-f(q)^{2}+\lambda e^{-q}-\epsilon=0
$$

The Taylor series about the origin

$$
f(q)=\sum_{j=0}^{\infty} f_{j} q^{j}, f_{0}=0
$$

converges in a neighbourhood of $q=0$ and the coefficients $f_{j}$ depend on $\epsilon$.

The main assumption of the RPM is that the roots of the Hankel determinants $H_{D}^{d}(\epsilon)=0$, with matrix elements $f_{j+j+d-1}, i, j=1,2, \ldots, D$, are suitable approximations to the eigenvalues of the Schrödinger equation (2) [2-11]. Here, $D=2,3, \ldots$ is the determinant dimension and $d=0,1, \ldots$ More precisely, we expect that there exists a sequence of roots $\epsilon^{[D, d]}$ of the Hankel determinants that converges to a given eigenvalue of that Schrödinger equation as $D$ increases. 


\section{Results and discussion}

Previous applications of the RPM showed that the rate of convergence of the Hankel sequences is remarkable for both real and complex eigenvalues [2-11]. However, in the case of the exponential potential (1) the Hankel sequences were found to converge to a result slightly different from the one given by equation (5) [9]. For example, Table1 1 shows Hankel sequences for $\lambda=0.5$ and $\lambda=2$ and the corresponding exact results obtained from the quantization condition (5)). Both Hankel sequences exhibit great convergence rate but they do not converge towards the expected result. We also appreciate that the disagreement between the exact and RPM eigenvalues increases as $\lambda$ decreases. In addition to the great convergence rate, the Hankel determinants exhibit clustering of roots about the limits of the sequences as $D$ increases, which is an indication of satisfactory convergence and meaningful result. However, those limits do not completely agree with the exact results given by (5) .

Fig. 1 shows values of $\epsilon(\lambda)$ calculated by means of the RPM and equation (51). As indicated above, the agreement between the RPM and exact results increases as $\lambda$ increases. For $\lambda \leq 0.4$ the RPM yields complex eigenvalues in spite of the fact that the exact ones are real (see, for example, Atabek et al [13] for a more detailed discussion of this behaviour). Moreover, it is well known that the roots $\nu$ of equation (5) tend to negative integers as $\lambda \rightarrow 0[13,14]$ but we clearly see that the RPM eigenvalues do not exhibit this behaviour. However, it is most interesting that both real and imaginary parts of the RPM eigenvalues give a reasonable picture of the behaviour of the exact ones for large and moderate values of $\lambda$ as shown in Fig. 1. In order to show the increasing agreement between the RPM and exact eigenvalue with $\lambda$ more clearly, Fig. 2 shows $\log \left|\epsilon^{\text {exact }}-\epsilon^{R P M}\right|$ as a function of $\lambda$.

The discrepancy between the RPM and exact eigenvalues just mentioned is interesting from a mathematical point of view, but it is not a serious drawback 
for physical purposes. It is well known that realistic potentials require much larger values of $\lambda$ than those in Fig. 1] [13]. If, for example we choose $\lambda=100$ the rate of convergence of the Hankel sequence is much greater than the one in Table 1 and the limit agrees with the exact result to at least 20 digits (see Table 2). Atabek et al [13] estimated the potential parameter for the ${ }^{2} \Sigma^{+}$ repulsive state of $\mathrm{BeH}$ to be $\Lambda \simeq 134$ that corresponds to $\lambda=4489$. In this case the rate of convergence of the Hankel sequence is even greater and we obtain the exact result to at least 20 digits with $D=15$ and $d=0$ as shown in Table 2. It is worth mentioning that for such large values of $\lambda$ we find it easier to obtain the complex energies by means of the RPM than from the roots of the Bessel function.

\section{Conclusions}

We have shown that the Hankel sequences converge to a wrong limit that is close to the resonances of the repulsive exponential potential. At present we do not know the reason for this discrepancy that decreases as the potential strength increases. However the RPM eigenvalue as function of the potential parameter follows the trend of the exact one. Besides, for realistic potential functions that fit the interaction between molecular fragments [13], the RPM provides remarkably accurate results and it is probably more accurate than other methods. Unfortunately we are not aware of results for such great values of the potential strength, probably because other methods do not provide the complex eigenvalues so efficiently as the RPM.

We should mention that the complex eigenvalues of the Schrödinger equation with the exponential potential (1) are not what physicist use to call resonances because the imaginary parts are too large. However, there has been some interest in their calculation with the purpose of reconstruction of the scattering amplitude from its poles $[13,14]$. 


\section{References}

[1] S. A. Rakityansky, S. A. Sofianos, and N. Elander, J. Phys. A 40 (2007) 1485714869.

[2] F. M. Fernández, Q. Ma, and R. H. Tipping, Phys. Rev. A 40 (1989) 6149-6153.

[3] F. M. Fernández, Q. Ma, and R. H. Tipping, Phys. Rev. A 39 (1989) 1605-1609.

[4] F. M. Fernández, Phys. Lett. A 166 (1992) 173-176.

[5] F. M. Fernández and R. Guardiola, J. Phys. A 26 (1993) 7169-7180.

[6] F. M. Fernández, Phys. Lett. A 203 (1995) 275-278.

[7] F. M. Fernández, J. Phys. A 28 (1995) 4043-4051.

[8] F. M. Fernández, J. Chem. Phys. 103 (1995) 6581-6585.

[9] F. M. Fernández, J. Phys. A 29 (1996) 3167-3177.

[10] F. M. Fernández, Phys. Rev. A 54 (1996) 1206-1209.

[11] F. M. Fernández, Chem. Phys. Lett 281 (1997) 337-342.

[12] S. T. Ma, Phys. Rev. 69 (1946) 668.

[13] O. Atabek, R. Lefebvre, and M. Jacon, J. Phys. B 15 (1982) 2689-2701.

[14] P. Midy, O. Atabek, and G. Oliver, J. Phys. B 26 (1993) 835-853. 
Table 1

Convergence of the Hankel sequences for two values of $\lambda$

\begin{tabular}{|c|c|c|}
\hline \multicolumn{3}{|c|}{$\lambda=0.5$} \\
\hline$D$ & $\operatorname{Re} \epsilon$ & $\operatorname{Im} \epsilon$ \\
\hline 10 & -0.70545054582805260895 & 0.26816598479688956569 \\
\hline 11 & -0.70545056661473611239 & 0.26816596401669675062 \\
\hline 12 & -0.70545056816101389381 & 0.26816596478162896896 \\
\hline 13 & -0.70545056810407142688 & 0.26816596486012868610 \\
\hline 14 & -0.70545056805626480670 & 0.26816596487152171555 \\
\hline 15 & -0.70545056805476309518 & 0.26816596487187249577 \\
\hline 16 & -0.70545056805499618233 & 0.26816596487156659341 \\
\hline 17 & -0.70545056805503003056 & 0.26816596487158015859 \\
\hline 18 & -0.70545056805502881287 & 0.26816596487157957123 \\
\hline 19 & -0.70545056805502843245 & 0.26816596487157970586 \\
\hline 20 & -0.70545056805502836237 & 0.26816596487157971713 \\
\hline Exact & -0.73985910415959609800 & 0.24527511363052010569 \\
\hline \multicolumn{3}{|c|}{$\lambda=2$} \\
\hline$D$ & $\operatorname{Re} \epsilon$ & $\operatorname{Im} \epsilon$ \\
\hline 10 & -0.66695560251365514674 & 1.6211700286378446183 \\
\hline 11 & -0.66695559703717080232 & 1.6211700273918467827 \\
\hline 12 & -0.66695559708065060107 & 1.6211700264998970342 \\
\hline 13 & -0.66695559710083543597 & 1.6211700264601278937 \\
\hline 14 & -0.66695559710850847182 & 1.6211700264500121775 \\
\hline 15 & $\begin{array}{c}8 \\
-0.66695559710929174524\end{array}$ & 1.6211700264509149572 \\
\hline
\end{tabular}


Table 2

Convergence of the Hankel sequences for large values of $\lambda$

\begin{tabular}{|c|c|c|}
\hline \multicolumn{3}{|c|}{$\lambda=100$} \\
\hline$D$ & $\operatorname{Re} \epsilon$ & $\operatorname{Im} \epsilon$ \\
\hline 5 & 71.535851840807002875 & 37.763655201763995538 \\
\hline 6 & 71.535265703486635320 & 37.763686464878442119 \\
\hline 7 & 71.535231112272632786 & 37.763673576025908553 \\
\hline 8 & 71.535229874257576952 & 37.763674377953883589 \\
\hline 9 & 71.535229860223483406 & 37.763674375421503193 \\
\hline 10 & 71.535229855328822688 & 37.763674374272030169 \\
\hline 11 & 71.535229855364554354 & 37.763674374318377973 \\
\hline 12 & 71.535229855364593976 & 37.763674374316154213 \\
\hline 13 & 71.535229855364798246 & 37.763674374316019149 \\
\hline 14 & 71.535229855364801073 & 37.763674374316016150 \\
\hline 15 & 71.535229855364801145 & 37.763674374316015925 \\
\hline 16 & 71.535229855364801148 & 37.763674374316015917 \\
\hline 17 & 71.535229855364801148 & 37.763674374316015916 \\
\hline \multicolumn{3}{|c|}{$\lambda=4489$} \\
\hline$D$ & $\operatorname{Re} \epsilon$ & $\operatorname{Im} \epsilon$ \\
\hline 5 & 4158.9571348913180566 & 530.18487292989576140 \\
\hline 6 & 4158.9534557328433593 & 530.18603523832850612 \\
\hline 7 & 4158.9533491082024576 & 530.18608879147133066 \\
\hline 8 & 4158.9533468989068860 & 530.18609014361891762 \\
\hline 9 & $\begin{array}{r}9 \\
4158.9533468479121394\end{array}$ & 530.18609015967783227 \\
\hline
\end{tabular}




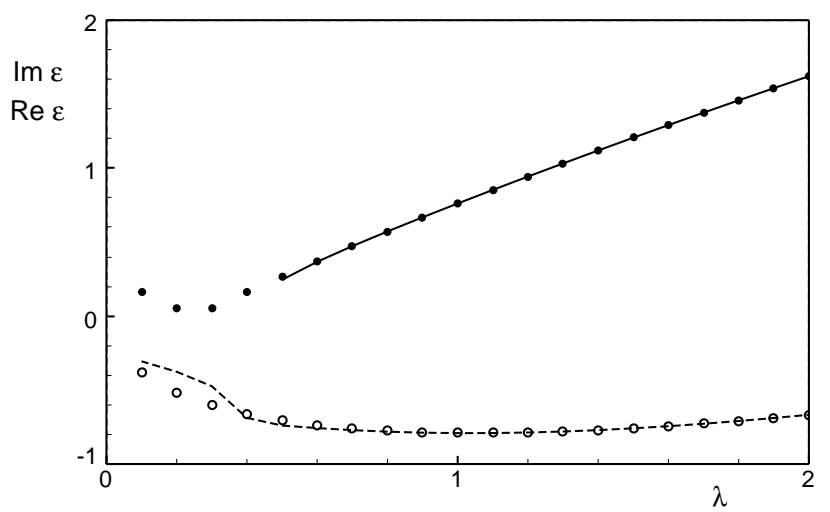

Fig. 1. Eigenvalues of the exponential potential: $\operatorname{Re} \epsilon^{R P M}$ (empty circles), $\operatorname{Im} \epsilon^{R P M}$ (solid circles), Re $\epsilon^{\text {exact }}$ (dashed line), Im $\epsilon^{\text {exact }}$ (solid line)

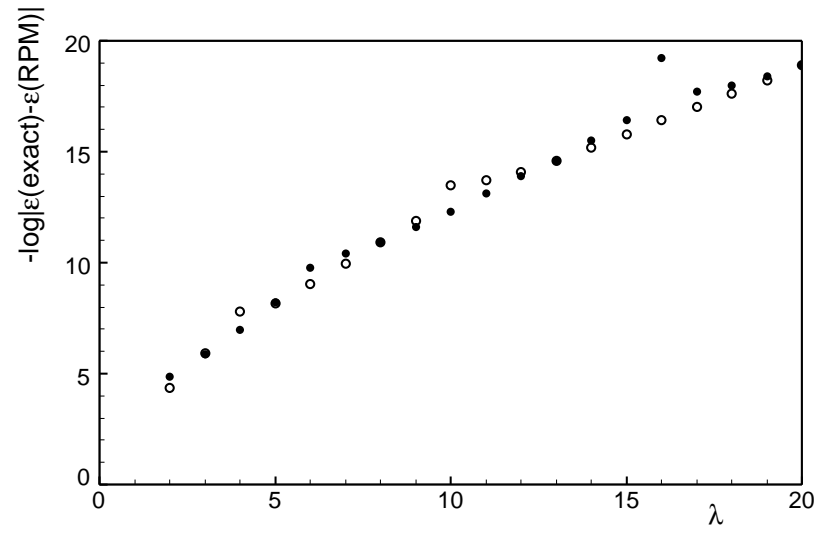

Fig. 2. $\log \left|\operatorname{Re} \epsilon^{\text {exact }}-\operatorname{Re} \epsilon^{R P M}\right|$ (empty circles) and $\log \left|\operatorname{Im} \epsilon^{\text {exact }}-\operatorname{Im} \epsilon^{R P M}\right|$ (solid circles) 